\title{
Gold Nanoparticle Coated Silicon Nitride chips for Intracellular Surface-Enhanced Raman Spectroscopy
}

\author{
Pieter C. Wuytens ${ }^{1,2}$, Alexey M. Yashchenok ${ }^{3}$, Ananth Z. Subramanian ${ }^{1}$, \\ Andre G. Skirtach ${ }^{2}$, Roel Baets ${ }^{1}$ \\ ${ }^{\text {I}}$ Photonics Research Group, Department of Information Technology, Ghent University - imec, B-9000 Ghent, Belgium \\ ${ }^{2}$ Department of Molecular Biotechnology, Ghent University, B-9000 Ghent, Belgium \\ ${ }^{3}$ Max-Planck Institute of Colloids and Interfaces, D-14476 Golm, Postdam, Germany \\ email: pieter.wuytens@intec.ugent.be
}

\begin{abstract}
Using surface-enhanced Raman spectroscopy on gold-nanoparticle-decorated silicon nitride chips, we monitor the release of dextran-rhodamin molecules from capsules inside living cells. This demonstrates the feasibility of using photonic chips for intracellular sensing at visible wavelengths.

OCIS codes: (180.5655) Raman microscopy, (170.1530) Cell analysis
\end{abstract}

\section{Introduction}

Raman spectroscopy provides a promising label-free alternative for the intracellular detection of molecules, which nowadays largely relies on fluorescence based microscopy. Although the latter technique benefits from the strong intrinsic signals and high selectivity of the labels, fluorescence microcopy has limitations due to the reliance on fluorophores. Raman spectroscopy can provide fingerprint specificity and allows multiplexed, label-free measurements, but is limited by its inherently low signal strength. Surface-enhanced Raman spectroscopy offers an attractive solution to obtain intracellular Raman spectra at reasonably low laser powers and short integration times. Most studies on intracellular SERS use colloidal gold nanoparticles for plasmonic enhancement [1]. However, this method suffers from a poor reproducibility as the enhancement changes in space and time due to movement of the particles and the formation of aggregates. A more reproducible signal can be obtained by using a fixed metal configuration. This approach is used in Tip-Enhanced Raman Spectroscopy, where a narrow gold-coated probe pierces through the cell membrane [2]. But piercing the cell membrane does not allow for long term monitoring of the cells and could impact viability of cells.

In this paper, we propose the use of lithography-fabricated Silicon Nitride ( $\mathrm{SiN}$ ) microchips - coated with gold nanoparticles - for intracellular SERS. These planar chips offer the advantage of a fixed metal configuration and a high surface to volume ratio. This provides not only a large measurement surface, but is also expected to have low cytotoxicity. A permanent incision of the cell membrane during measurements is avoided. We demonstrate the potential of this approach in a drug delivery application by monitoring the laser-triggered release of dextran-linked rhodamin molecules from a polymeric-shell capsule. In the near future, the use of SiN will allow us to design more complex photonic chips for intracellular sensing at visible or near-infrared wavelengths, i.e. in the so-called "biofriendly" spectral window.

\section{Experiments}

Micrometer sized SiN chips were fabricated by depositing $200 \mathrm{~nm}$ layer of PECVD SiN on a Si wafer. In this layer, individual chips were defined through contact lithography, followed by reactive ion etching. Next the underlying silicon layer was wet etched using potassium hydroxide, during which ultrasound was applied to efficiently detach the chips from the substrate. After wet etching, the detached SiN chips were collected in water via centrifugation. Finally the chips were coated by adsorption of positively charged gold nanoparticles. Scanning electron microscopy images show a good coverage of the SiN chips with gold nanoparticles (Fig. 1). Polyelectrolyte capsules containing dextran - rhodamin molecules were fabricated using a method described earlier [3]. The polyelectrolyte shells of the capsules contain gold nanoparticles, which opens the capsules due to localized heating around the nanoparticles under infrared laser light. This laser-triggered opening allows us to release molecules from inside the cell at a specified time and location.

Both SiN chips and capsules were inserted into a normal human dermal fibroblast (NHDF) cell culture using a method based on electroporation. Although further viability studies are required, cells division still occurred with cells having incorporated capsules and chips inside. After uptake, the intracellular capsules were opened using a 
focused laser beam under a commercial Raman microscope at a power density $30 \mathrm{~mW} \mu \mathrm{m}^{-2}$, upon which dextranrhodamin molecules were released from the capsule. This method enables to instantaneously monitor the release of the molecules through their Raman spectrum, as lowering the power density $\left(2 \mathrm{~mW} \mu \mathrm{m}^{-2}\right)$ allows to measure SERS spectra on the adjacent SiN chips in the same cell. Fig. 2(a) shows a transmission image of a cell containing three capsules and two disk-shaped SiN chips (dashed red arrows). This image was taken after the laser-triggered opening of the lowest capsule (yellow arrow). The release of the red-fluorescent dextran-rhodamin molecules is confirmed by confocal fluorescence microscopy (Fig. 2(b)), showing the leakage of the molecules out of the capsule. Fig. 2(c) compares the reference spectrum of the loaded molecules (measured in a $1 \mathrm{mg} / \mathrm{ml}$ solution, $30 \mathrm{~mW}$ on sample and 4 minute integration time) with a SERS spectrum collected on top of the SiN chips $(2 \mathrm{~mW}, 0.5 \mathrm{sec}$ integration time) after capsule release. The presence of other peaks can be explained by the complex environment of the cell. It is important to notice that the imaged cell stayed alive during the entire experiment.

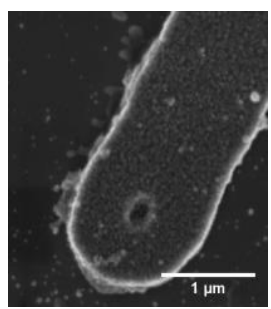

Fig 1. SEM image of a rectangular-shaped gold-covered SiN particle (thickness 200 $\mathrm{nm})$

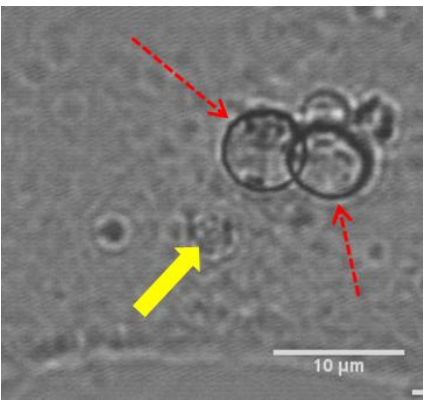

(a)

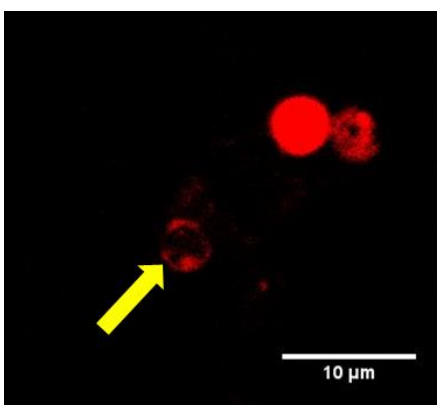

(b)

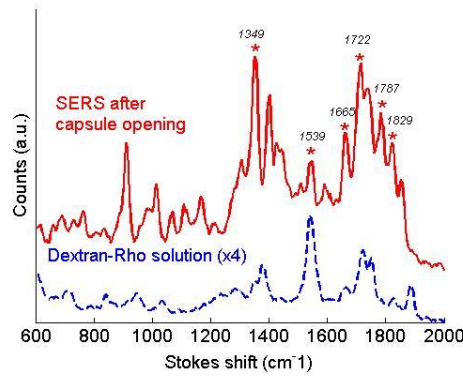

(c)

Fig. 2: (a) Human fibroblast cell containing three dextran-rhodamin loaded capsules and two gold coated SiN chips (red dashed arrows).(b) Fluorscence signal after opening one capsule (yellow arrow) (c) SERS spectra measured on top of the SiN chips after capsule release (red) and reference spectrum of dextran-rhodamin (blue). Stars indicate corresponding Raman lines

\section{Conclusion and future perspective}

We demonstrated the possibility to use SiN chips for intracellular SERS experiments, and developed a method for intracellular delivery and immediate Raman detection on the same microscope. In a model experiment, dextranrhodamin dyes were detected through their SERS spectrum, the molecule release was confirmed by confocal fluorescence microscopy. In later stages, our approach can be extended towards monitoring the release of (nonfluorescent) drugs.

\section{References}

[1] K. Kneipp, Y. Wang et al, "Single Molecule Detection Using Surface-Enhanced Raman Scattering (SERS)", Phys. Rev. Letters 78, 16671670 (1997)

[2] E. Vitol, Z. Orynbayeva et al., "Nanoprobes for intracellular and single cell surface-enhanced Raman spectroscopy (SERS)", J. Raman Spectrosc. 43-7, 1371-1377

[3] A. Skirtach, C. Dejugnat et al., "The role of metal nanoparticles in remote release of encapsulated materials", Nano Letters 5-7, 1371-1377 (2005)

[4] A. Subramanian et. al., "Low-loss singlemode PECVD silicon nitride photonic wire waveguides for 532-900 nm wavelength window fabricated within a CMOS pilot line", IEEE Photonics Journal 5, 2202809 (2013) 